\title{
Three-dimensional atomic imaging of crystalline nanoparticles
}

\author{
Sandra Van Aert ${ }^{1}$, Kees J. Batenburg ${ }^{2,3}$, Marta D. Rossell ${ }^{4}$, Rolf Erni ${ }^{5}$ \& Gustaaf Van Tendeloo ${ }^{1}$
}

\begin{abstract}
Determining the three-dimensional (3D) arrangement of atoms in crystalline nanoparticles is important for nanometre-scale device engineering and also for applications involving nanoparticles, such as optoelectronics or catalysis. A nanoparticle's physical and chemical properties are controlled by its exact 3D morphology, structure and composition ${ }^{1}$. Electron tomography enables the recovery of the shape of a nanoparticle from a series of projection images ${ }^{2-4}$. Although atomic-resolution electron microscopy has been feasible for nearly four decades, neither electron tomography nor any other experimental technique has yet demonstrated atomic resolution in three dimensions. Here we report the 3D reconstruction of a complex crystalline nanoparticle at atomic resolution. To achieve this, we combined aberration-corrected scanning transmission electron microscopy ${ }^{5-7}$, statistical parameter estimation theory ${ }^{8,9}$ and discrete tomography ${ }^{10,11}$. Unlike conventional electron tomography, only two images of the target-a silver nanoparticle embedded in an aluminium matrix-are sufficient for the reconstruction when combined with available knowledge about the particle's crystallographic structure. Additional projections confirm the reliability of the result. The results we present help close the gap between the atomic resolution achievable in two-dimensional electron micrographs and the coarser resolution that has hitherto been obtained by conventional electron tomography.
\end{abstract}

High-angle annular dark field scanning transmission electron microscopy (HAADF STEM) is an imaging technique in which a focused electron probe is scanned across an electron-transparent sample ${ }^{12}$. Using an annular-shaped high-angle detector behind the sample, the signal is dominated by Rutherford and thermal diffuse scattering. When applied to a nanocrystal in zone-axis orientation, the HAADF signal approximately scales with the square of the atomic number $Z$ and with the thickness of the sample $\mathrm{e}^{13-15}$. By using aberration-corrected probe forming optics ${ }^{5-7}$, a resolution of the order of 50 picometres can nowadays be demonstrated ${ }^{16}$. Therefore, it is generally believed that aberration-corrected HAADF STEM has the potential to achieve atomic resolution in three dimensions. Electron tomography is the most common approach used to reconstruct nanomaterials in three dimensions. The $3 \mathrm{D}$ reconstruction is computed from a tilt series of projection images acquired while rotating the sample. Spatial resolution for the reconstruction is around one cubic nanometre ${ }^{2-4}$, limiting its use for attaining atomic resolution. Another potential technique with which to obtain 3D structure information is 'depth-sectioning', in which a sample is optically sliced by changing the objective lens focus ${ }^{17}$. Single atoms can be visualized using this technique ${ }^{18}$, yet $3 \mathrm{D}$ reconstructions at atomic resolution have not been demonstrated because the depth resolution is insufficient to resolve interatomic distances along the optical axis. Discrete tomography, a reconstruction approach that exploits prior knowledge about the discrete nature of atoms and their lattice structure, has been proposed as a promising technique for atomic resolution tomography ${ }^{10,11}$, but only simulation results have been presented. Li et al. ${ }^{19}$ showed a 3D structure analysis of a gold cluster from one single HAADF STEM image. However, this approach required strong a priori knowledge in combination with image simulations of regularly shaped models. Previous attempts have mostly focused on the technique and image acquisition, whereas the interpretation of the images was oversimplified, not taking into account the detailed probe characteristics ${ }^{20-22}$ and the statistical nature of the experimental data. Here, we combine aberration-corrected HAADF STEM carried out under low voltage conditions with modelbased statistical parameter estimation ${ }^{8,9}$ and discrete tomography ${ }^{10,11}$ to obtain a full atomic-scale $3 \mathrm{D}$ reconstruction of an embedded nanoparticle.

A binary alloy consisting of $\mathrm{Al}$ with 3 at.\% $\mathrm{Ag}$ was homogenized at $550{ }^{\circ} \mathrm{C}$ and quenched in ice-brine. During quenching, small Ag-rich clusters are formed which are fully coherent with the surrounding Alrich matrix. This is clear from the electron diffraction patterns in Fig. la and d, from which it can not only be deduced that the structure of matrix and particle is face-centred cubic, but also that there is no lattice mismatch involved between matrix and particle (spot-splitting of the reflections, even far from the origin, is absent). The embedded clusters, with diameters slightly exceeding $2 \mathrm{~nm}$, consist of nearly $100 \% \mathrm{Ag}$ (refs 23 and 24). Electron-transparent foils with a measured thickness of about $12 \mathrm{~nm}$ were investigated in an electron microscope equipped with aberration correctors in the probe- and image-forming optical parts and operated at $80 \mathrm{kV}$ (ref. 25). Although the resolution is better at higher acceleration voltages, operating the microscope at $80 \mathrm{kV}$ guarantees the integrity of the nanoparticles during the acquisition of the data ${ }^{26}$. Moreover, the particles, which are $2-3 \mathrm{~nm}$ in diameter, are fully contained in the depth of field of the electron probe, which is between 7 and $10 \mathrm{~nm}^{27}$. High-resolution HAADF STEM images of nanosized Ag clusters embedded in the Al matrix viewed along the $[10 \overline{1}]$ and [100] zone-axes are shown in Fig. 1a and d, respectively. The white boxes enclose the same particle in both orientations.

A crucial step towards quantitative $3 \mathrm{D}$ structure determination of a nanoparticle at atomic resolution is the ability to count the number of atoms in each projected column. If one repeats this procedure reliably for several viewing directions, one will be able to reconstruct the $3 \mathrm{D}$ space using discrete tomography ${ }^{10,11}$. We used a recent quantitative method to analyse HAADF STEM images, in which atomic columns with a difference in (average) atomic number of only three could be identified ${ }^{9}$. This model-based method accounts for the tails of the scanning probe $e^{20-22}$ (see Supplementary Information). Refined models, describing the contrast of the nanoparticle enclosed in the white boxes of Fig. 1a and d, are shown in Fig. $1 \mathrm{~b}$ and e, respectively. The excellent agreement with the experimental contrast demonstrates the quality of the physics-based model used (see Supplementary Figs 1 and 2). Although dynamical scattering has not explicitly been taken into account, so-called cross-talk is shown to be negligible for the images in the $[10 \overline{1}]$ and [100] zone-axes (see Supplementary Information and Supplementary Fig. 3). (This effect, which seriously complicates the analysis, refers to transfer of probe intensity from one column to another column.) 

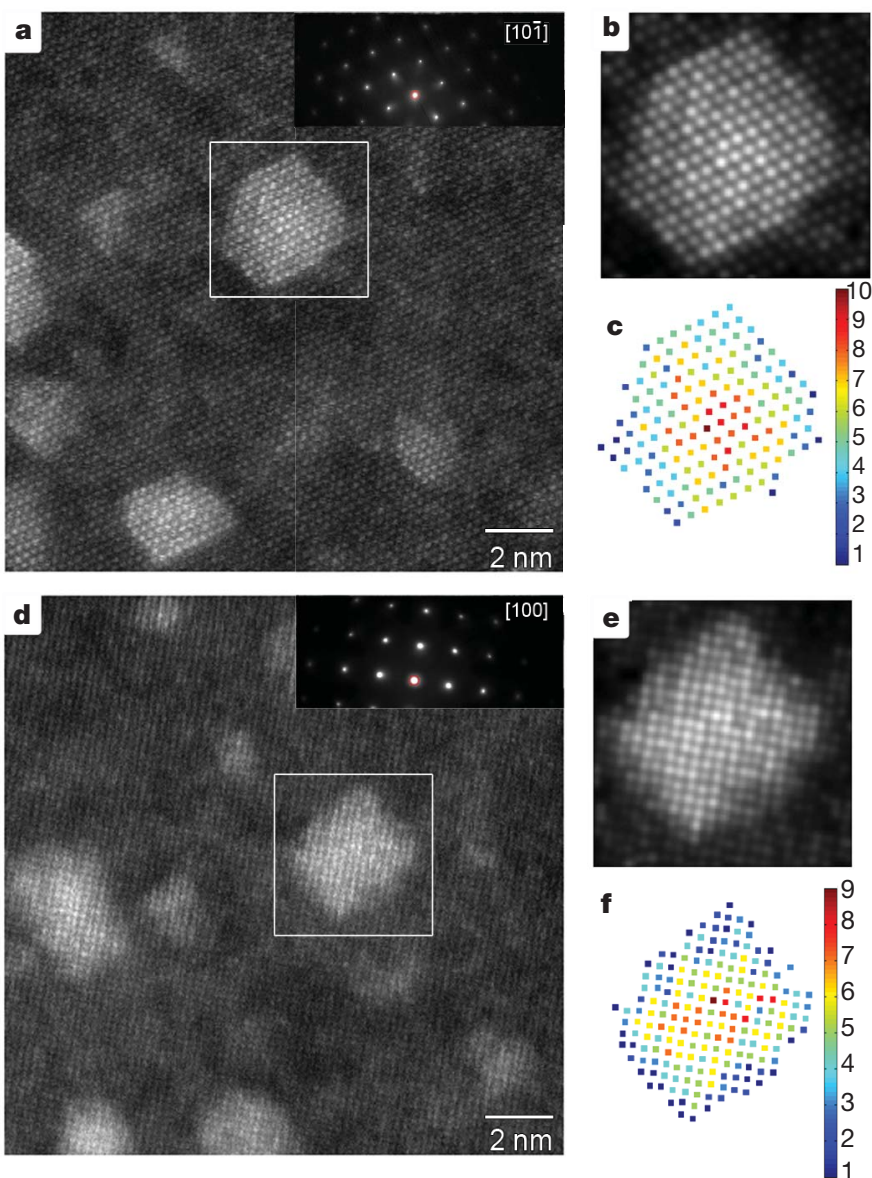

Figure $1 \mid$ Quantification of HAADF STEM images. a, Experimental HAADF STEM image of nanosized Ag clusters embedded in an Al matrix in $[10 \overline{1}]$ zone-axis orientation, together with the corresponding electron diffraction pattern. $\mathbf{b}$, Refined model of the boxed region in a. c, Number of Ag atoms per column. d, Experimental HAADF STEM image in [100] zone-axis orientation, together with the corresponding electron diffraction pattern. e, Refined model of the boxed region in $\mathbf{d}$. $\mathbf{f}$, Number of Ag atoms per column.

Next, the scattered intensities can be computed ${ }^{9}$. These intensities scale with the average atomic number $Z$, allowing one to distinguish columns containing a certain amount of $\operatorname{Ag}(Z=47)$ from pure $\mathrm{Al}$ $(Z=13)$ columns. For the nanoparticle viewed along the $[10 \overline{1}]$ zoneaxis, these columns are indicated by red markers in Fig. 2a. The corresponding scattered intensities are shown in the histogram in Fig. $2 b$. Because the thickness of the sample can be assumed to be constant over the particle area, substitution of an $\mathrm{Al}$ atom by an $\mathrm{Ag}$ atom leads to an increase of the estimated intensity. An experimental histogram, however, shows broadened-rather than discrete-peaks owing to a combination of experimental detection noise, the surrounding aluminium matrix and residual instabilities. To determine the number of significant peaks and their positions, a statistics-based approach is proposed. The intensities are regarded as independent statistical draws from a so-called Gaussian mixture model ${ }^{28}$. This model, defined as a superposition of Gaussians, describes the probability that a specific intensity value will be observed. To determine the number of significant peaks of this model, the Integrated Classification Likelihood criterion was used because it outperforms other order-selection criteria $^{28}$. In Fig. $2 c$, this criterion has been evaluated for an increasing number of peaks, clearly showing a minimum at ten. This indicates the presence of ten significant peaks in the histogram of Fig. 2b. Their positions, amplitudes and width have been estimated using the wellknown expectation maximization algorithm ${ }^{28}$. Its aim is to optimize the likelihood that the given intensities are generated by a mixture of Gaussians. The black solid curve in Fig. 2b shows the estimated mixture model; the individual Gaussian components are shown by means of dashed curves. Even though this has not been assumed a priori in the model, it is clear from Fig. $2 \mathrm{~b}$ that the Gaussians are equidistantly separated. This means that the estimated mean intensity values, shown by black dots, linearly increase with the number of atoms in a column ${ }^{13,14}$ (see Supplementary Fig. 4). Next, the estimated mixture model can be used to identify the number of atoms in a particular column, as shown in Fig. 1c. The same counting procedure has been applied for the particle viewed along the [100] zone-axis, resulting in Fig. If

Using the atom counting results shown in Fig. 1c and f, discrete tomography was used to obtain a $3 \mathrm{D}$ reconstruction from these two projections. In general, two projections are insufficient to determine the $3 \mathrm{D}$ structure uniquely, because there may be many atom configurations leading to the same projections. We therefore incorporated here the following particle-specific knowledge, which is justified ${ }^{23,24,26}$ or could be verified: (1) the atoms are situated on a face-centred-cubic lattice, (2) the particle contains no holes, (3) each of the interior 2D slices (in the $x / y / z$ direction) that are at least two slices away from the particle boundary should be connected in 2D and contain no holes. We note that this a priori knowledge depends on the structure and configuration of interest. For a free-standing crystalline nanoparticle of any shape, even concave or containing holes, the same elegant procedure can still be applied to resolve the atoms in three dimensions.

Using a customized implementation of the simulated annealing algorithm for optimization ${ }^{29}$, the reconstruction shown in Fig. 3a and in Supplementary Movie 1 was computed. Because the optimization algorithm is stochastic, it can be run repeatedly, each time yielding a reconstruction that may be different from the previous one. In 16 independent reconstruction runs, we observed a maximum difference
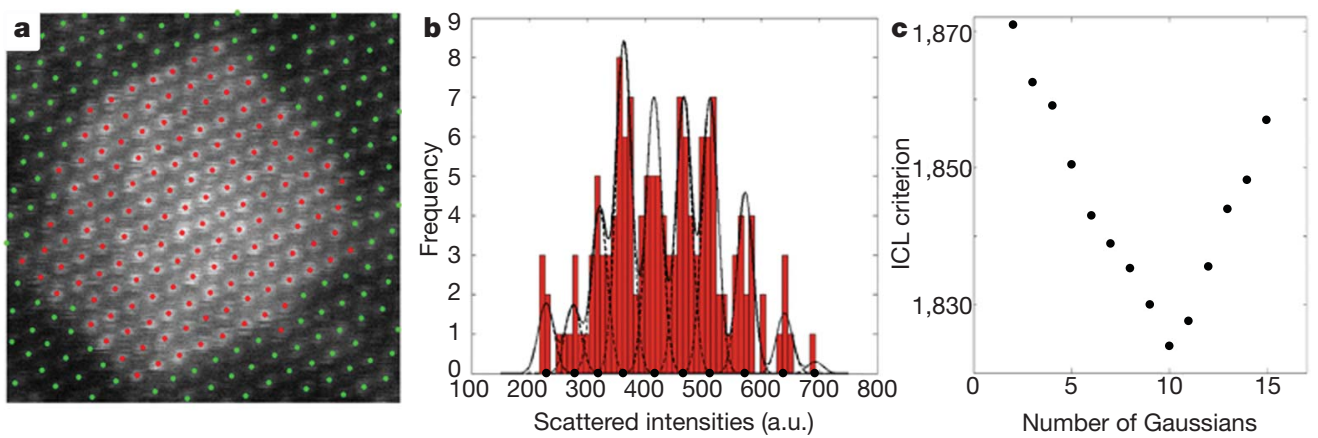

Figure $2 \mid$ Analysis of scattered intensities. a, Magnification of the boxed region in Fig. 1a. Columns containing Ag are indicated by red markers placed at the estimated positions. b. Histogram of scattered intensities of the Ag columns. The black solid curve shows the estimated mixture model; the individual components are shown as dashed curves. c, The Integrated Classification Likelihood criterion evaluated as a function of the number of Gaussians in a mixture model. 

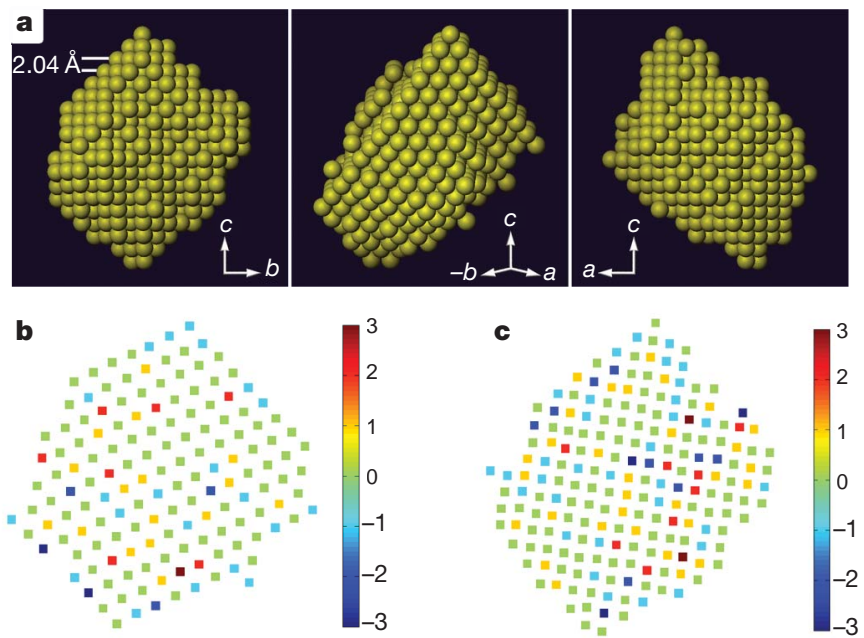

Figure $3 \mid$ Three-dimensional reconstruction. a, The computed 3D reconstruction of the $\mathrm{Ag}$ nanocluster viewed along three different directions. b, c, Difference between the computed atom counts for the reconstructed particle and the atom counts determined by the experiment in $[10 \overline{1}](\mathbf{b})$ and in [100] (c) zone-axis orientation, respectively.

in the positioning of only 41 atoms on a total of 784 atoms. This indicates that the reconstruction is well determined by the projection data. Figure $3 b$ and $c$ shows the difference between the computed atom counts for the reconstructed crystal and the atom counts determined by the experiment. Although the match is not perfect owing to experimental errors, it is bounded by a maximum column difference of three, and far less on average.

The excellent match between the $3 \mathrm{D}$ reconstruction and the experimental data are illustrated in Fig. 4. Figure 4a-d shows micrographs of the particle along the $[10 \overline{1}],[100],[4 \overline{1} \overline{1}]$ and $[2 \overline{1} \overline{1}]$ zone-axes, together with the corresponding snapshots (Fig. $4 \mathrm{e}-\mathrm{h}$ ) of Supplementary Movie 2. Owing to the limited resolution at $80 \mathrm{kV}$ and cross-talk between closely spaced atomic columns, the micrographs in the $[2 \overline{1} \overline{1}]$ and $[4 \overline{1} \overline{1}]$ zone-axes could not be used in the atom count procedure. Nonetheless, the extra information from the $[2 \overline{1} \overline{1}]$ and $[4 \overline{1} \overline{1}]$ projections provides confidence about the validity of the reconstructed $3 \mathrm{D}$ particle's shape and the reliability of this new method. The validity of the reconstruction and the match with the underlying data are particularly convincing considering the potential experimental errors that could lead to errors in the count data for projected atom columns. To obtain a site-specific confidence estimate for each atom in the reconstruction, a second series of 20 independent reconstructions was computed in which the observed atom counts were perturbed, taking into
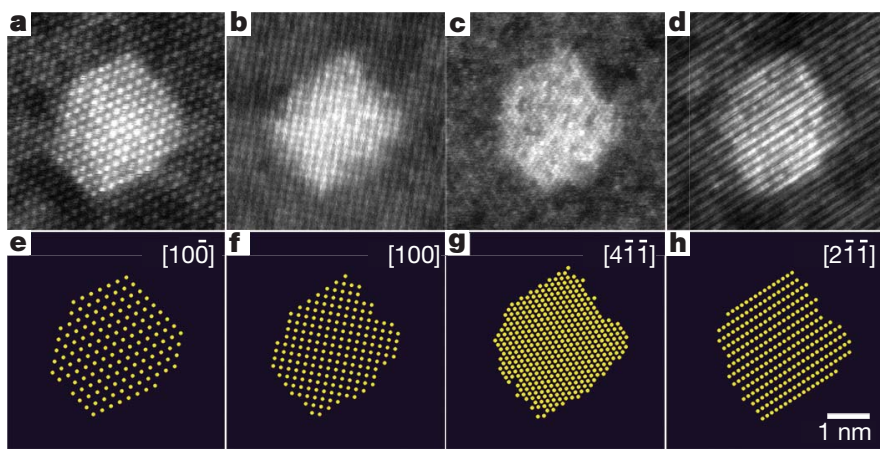

Figure $4 \mid$ Comparison of experimental images with projected 3D reconstructions. a-d, Images along $[10 \overline{1}],[100],[4 \overline{1} \overline{1}]$ and $[2 \overline{1} \overline{1}]$. $\mathbf{e}-\mathbf{h}$, Projections of the $3 \mathrm{D}$ reconstruction along the same directions. The experimental images along the $[4 \overline{1} \overline{1}]$, and $[2 \overline{1} \overline{1}]$ directions, which were not used for the $3 \mathrm{D}$ reconstruction, confirm the shape of the reconstructed $3 \mathrm{D}$ model. account that there is a probability of $5 \%$ of miscounting the total number of atoms in a column with one atom. This probability follows directly from the overlap of the Gaussian components shown in Fig. 2b. The probability of assigning a wrong number of atoms indeed increases with increasing overlap. By analysing the discrepancies between the 20 reconstructions thus obtained and the model in Fig. 3, a site-specific confidence map can be constructed (see the Supplementary Information, Supplementary Figs 5 and 6, and Supplementary Movie 3). The results indicate that over all atoms in our $3 \mathrm{D}$ reconstruction, the probability of wrongly assigning an atom at a specific site is only $3 \%$, indicating a high level of confidence for the reconstruction.

In conclusion, we have demonstrated a comprehensive approach for three-dimensional atomic imaging: the combination of HAADF STEM, atom counting using a thorough model-based statistical analysis and $3 \mathrm{D}$ reconstruction by applying discrete tomography from only two projected images. The close match of the $3 \mathrm{D}$ reconstruction with the atom counts and particle shape information based on experimental data provides confidence that the proposed method represents a reliable routine for atomic resolution tomography of crystalline nanoparticles. Using nanosized Ag clusters embedded in an Al matrix we have demonstrated the strength of this method. Our results reveal the great potential of this new technique for various applications regarding the atomic characterization of all kinds of complex nanometre-scale structured materials, particularly nanoparticles and nanocavities.

\section{METHODS SUMMARY}

Specimen preparation. A binary alloy consisting of $\mathrm{Al}$ with 3 at.\% $\mathrm{Ag}$ was prepared in an electric arc-furnace under an $\mathrm{Ar}$ atmosphere of 15 torr from pure $\mathrm{Al}$ (99.999\%) and $\mathrm{Ag}_{2} \mathrm{Al}$; it was homogenized for $3 \mathrm{~h}$ at $550^{\circ} \mathrm{C}$ and quenched in icebrine. During quenching small Ag-rich clusters are formed. Electron-transparent films were produced by mechanical polishing followed by electrolytic thinning. Using the EELS-based $t / \lambda$ method $^{30}$, a specimen thickness of about $12 \mathrm{~nm}$ was measured.

STEM imaging. Electron microscopy was carried out using the TEAM0.5 microscope operated at $80 \mathrm{kV}$ installed at the National Center for Electron Microscopy (NCEM) at the Lawrence Berkeley National Laboratory (LBNL). The images were recorded on a 3-mm-diameter specimen mounted to a double-tilt holder in a conventional compu-stage that enables a tilting range of $\pm 20-25^{\circ}$ in combination with the ultrahigh-resolution pole-piece lens employed. A probe semi-convergence angle of $25.7 \mathrm{mrad}$ was used. Hence, the (geometrical) depth of field is between 6 and $7 \mathrm{~nm}$. The effects of chromatic defocus spread even enlarge the depth of field to about $10 \mathrm{~nm}$ (ref. 27). The HAADF detector was set to collect electrons scattered between 70 and $490 \mathrm{mrad}$. This detector setting guarantees that the collected signal results in an approximately incoherent atomic-number contrast STEM image.

Received 6 May; accepted 3 December 2010.

Published online 2 February 2011.

1. Olson, G. B. Designing a new material world. Science 288, 993-998 (2000).

2. Arslan, I., Yates, T. J. V., Browning, N. D. \& Midgley, P. A. Embedded nanostructures revealed in three dimensions. Science 309, 2195-2198 (2005).

3. Midgley, P. A. \& Dunin-Borkowski, R. E. Electron tomography and holography in materials science. Nature Mater. 8, 271-280 (2009).

4. Batenburg, K. J. et al. 3D imaging of nanomaterials by discrete tomography. Ultramicroscopy 109, 730-740 (2009).

5. Haider, M. et al. Electron microscopy image enhanced. Nature 392, 768-769 (1998).

6. Batson, P. E., Dell by, N. \& Krivanek, O. L. Sub-ångstrom resolution using aberration corrected electron optics. Nature 418, 617-620 (2002)

7. Müller, H., Uhlemann, S., Hartel, P. \& Haider, M. Advancing the hexapole $\mathrm{C}_{\mathbf{s}^{-}}$ corrector for the scanning transmission electron microscope. Microsc. Microanal. $12,442-455$ (2006)

8. den Dekker, A. J., Van Aert, S., van den Bos, A. \& Van Dyck, D. Maximum likelihood estimation of structure parameters from high resolution electron microscopy images. Part I: a theoretical framework. Ultramicroscopy 104, 83-106 (2005).

9. Van Aert, S. etal. Quantitative atomic resolution mapping using high-angle annular dark field scanning transmission electron microscopy. Ultramicroscopy 109 , 1236-1244 (2009).

10. Batenburg, K. J. A network flow algorithm for reconstructing binary images from discrete x-rays. J. Math. Imaging Vision 27, 175-191 (2007).

11. Jinschek, J. R. et al. 3-D reconstruction of the atomic positions in a simulated gold nanocrystal based on discrete tomography: Prospects of atomic resolution electron tomography. Ultramicroscopy 108, 589-604 (2008). 
12. Crewe, A. V., Wall, J. \& Welter, L. M. A high-resolution scanning transmission electron microscope. J. Appl. Phys. 39, 5861-5868 (1968).

13. Hartel, P., Rose, H. \& Dinges, C. Conditions and reasons for incoherent imaging in STEM. Ultramicroscopy 63, 93-114 (1996)

14. Nellist, P. D. \& Pennycook, S. J. The principles and interpretation of annular darkfield Z-contrast imaging. Adv. Imaging Electron Phys. 113, 147-203 (2000).

15. Krivanek, O. L. et al. Atom-by-atom structural and chemical analysis by annula dark-field electron microscopy. Nature 464, 571-574 (2010).

16. Erni, R., Rossell, M. D., Kisielowski, C. \& Dahmen, U. Atomic-resolution imaging with a sub-50-pm electron probe. Phys. Rev. Lett. 102, 096101 (2009).

17. Pennycook, S. J., Lupini, A. R., Borisevich, A. Y., Peng, Y. \& Shibata, N. 3D atomic resolution imaging through aberration-corrected STEM. Microsc. Microanal. 10 (Suppl. 1.2), 1172-1173 (2004)

18. van Benthem, K. et al. Three-dimensional ADF imaging of individual atoms by through-focal series scanning transmission electron microscopy. Ultramicroscopy 106, 1062-1068 (2006).

19. Li, Z. Y. et al. Three-dimensional atomic-scale structure of size-selected gold nanoclusters. Nature 451, 46-48 (2008).

20. Hillyard, S. \& Silcox, J. Detector geometry, thermal diffuse-scattering and strain effects in ADF STEM imaging. Ultramicroscopy 58, 6-17 (1995).

21. Klenov, D. O., Zide, J. M., Zimmerman, J. D., Gossard, A. C. \& Stemmer, S. Interface atomic structure of epitaxial ErAs layers on (001) $\ln _{0.53} \mathrm{Ga}_{0.47} \mathrm{As}$ and $\mathrm{GaAs}$. Appl. Phys. Lett. 86, 241901 (2005)

22. Dwyer, C., Erni, R. \& Etheridge, J. Measurement of effective source distribution and its importance for quantitative interpretation of STEM images. Ultramicroscopy 110, 952-957 (2010)

23. Dubey, Ph. A., Schönfeld, B. \& Kostorz, G. Shape and internal structure of GuinierPreston zones in Al-Ag. Acta Metall. Mater. 39, 1161-1170 (1991)

24. Malik, A., Schonfeld, B., Kostorz, G. \& Pedersen, J. S. Microstructure of GuinierPreston zones in Al-Ag. Acta Mater. 44, 4845-4852 (1996).

25. Kisielowski, C. et al. Detection of single atoms and buried defects in three dimensions by aberration-corrected electron microscope with 0.5-angstrom information limit. Microsc. Microanal. 14, 469-477 (2008).
26. Erni, R, Heinrich, H. \& Kostorz, G. On the internal structure of Guinier-Preston zones in Al-3 at.\% Ag. Phil. Mag. Lett. 83, 599-609 (2003).

27. Xin, H. L. \& Muller, D. A. Aberration-corrected ADF-STEM depth sectioning and prospects for reliable 3D imaging in S/TEM. J. Electron Microsc. 58, 157-165 (2009).

28. McLachlan, G. \& Peel, D. Finite Mixture Models (eds Shewhart, W. A. \& Wilks, S. S.) (Wiley Series in Probability and Statistics, John Wiley \& Sons, 2000).

29. Kirkpatrick, S., Gelatt, C. D. \& Vecchi, M. P. Optimization by simulated annealing, Science 220, 671-680 (1983)

30. Malis, T., Cheng, S. C. \& Egerton, R. F. EELS log-ratio technique for specimenthickness measurement in the TEM. J. Electron Microsc. Tech. 8, 193-200 (1988).

Supplementary Information is linked to the online version of the paper at www.nature.com/nature.

Acknowledgements Part of this work was performed at the National Center for Electron Microscopy (LBNL) which is supported by the Office of Science, Office of Basic Energy Sciences of the US Department of Energy under contract number

DE-AC02-05CH1 1231. Financial support from the European Union for the Framework 6 programme under a contract for an Integrated Infrastructure Initiative (reference 026019 ESTEEM) is acknowledged.

Author Contributions S.V.A. developed and applied a method of counting the number of atoms. K.J.B. reconstructed the nanoparticle in three dimensions. M.D.R. and R.E. prepared the sample and recorded the experimental images. G.V.T. advised on the methodology, the interpretation and on the paper. All the authors read and commented on the paper.

Author Information Reprints and permissions information is available at www.nature.com/reprints. The authors declare no competing financial interests. Readers are welcome to comment on the online version of this article at www.nature.com/nature. Correspondence and requests for materials should be addressed to S.V.A. (sandra.vanaert@ua.ac.be). 